\title{
改性活性炭负载 $\mathrm{FeCl}_{3}$ 催化一锅法合成多芳基咪唑
}

\author{
肖立伟* 高红杰 孔 洁 刘光仙李玲玲 段敬丹
}

(廊坊师范学院化学与材料科学学院 廊坊 065000)

\begin{abstract}
摘要 制备了一种新型改性活性炭负载氯化铁催化剂 $\left(\mathrm{MAC}-\mathrm{FeCl}_{3}\right)$, 通过红外光谱及等离子体发射光谱进行了表征. 在 $\mathrm{MAC}-\mathrm{FeCl}_{3}$ 催化下, 以乙酸铵为氮源, 苯偶酰与芳醛以及伯胺作用, 一锅法合成了一系列 2,4,5-三芳基咪唑和 1,2,4,5-四芳基咪唑. 催化剂后处理简单，重复使用 4 次仍保持较高活性. 该方法收率较高、对环境友好、操作简单.

关键词＼cjkstart改性活性炭负载 $\mathrm{FeCl}_{3} ; 2,4,5$-三芳基咪㟇; 1,2,4,5-四芳基咪唑; 一锅法; 异相催化
\end{abstract}

\section{One Pot Synthesis of Multiaryl Imidazoles Catalyzed by Modified Active Carbon Surported $\mathrm{FeCl}_{3}$}

\author{
Xiao, Liwei* Gao, Hongjie Kong, Jie Liu, Guangxian Li, Lingling Duan, Jingdan \\ (Faculty of Chemistry and Material Science, Langfang Teachers College, Langfang 065000)
}

\begin{abstract}
A novel modified active carbon surported ferric chloride catalyst $\left(\mathrm{MAC}-\mathrm{FeCl}_{3}\right.$ ) has been developed, which has been characterized by IR and ICP. $\mathrm{MAC}-\mathrm{FeCl}_{3}$ exhibited excellent catalytic activities for one-pot synthesis of 2,4,5-triaryl imidazoles and 1,2,4,5-tetraaryl imidazoles from ammonium acetate, benzil, aromatic aldehydes or primary amines. The catalyst could be recycled easily and the activity of catalyst maintains stability after reused for 4 times, which makes this methodology has the advantages of good yields, less pollution and simple work-up.
\end{abstract}

Keywords modified active carbon surported $\mathrm{FeCl}_{3}$; 2,4,5-triaryl imidazoles; 1,2,4,5-tetraaryl imidazoles; one-pot method; heterogeneous catalysis

多取代咪唑类化合物包括三取代咪唑和四取代咪 唑，其中常见的是 2,4,5-三取代咪唑及 1,2,4,5-四取代咪 唑. 这些含氮杂环及其衍生物具有很强的生理活性, 可 作为 $\mathrm{p} 38$ MAP、蛋白激酶抑制剂、钻拮抗剂、强心剂、 抗肿瘤药物等, 在医药领域有广泛的应用 ${ }^{[1,2]}$. 此外, 以 咪唑盐类为主体的离子液体, 因其优良的理化性能已经 引起人们的极大兴趣, 在有机合成、催化、萃取、采矿 等领域显示出良好的应用前景 ${ }^{[3]}$. 制备 2,4,5-三取代咪 唑及 1,2,4,5-四取代咪唑类化合物的反应条件类似, 通 常由邻二酮、醛或胺为原料, 醋酸铵等为氮源通过多组 分一锅法合成. 采用的催化剂既有均相催化剂如 $\mathrm{KH}_{2} \mathrm{PO}_{4}{ }^{[4]} 、 \mathrm{ZrOCl}_{2} \cdot 8 \mathrm{H}_{2} \mathrm{O}^{[5]}$ 、杂多酸 ${ }^{[6]} 、\left(\mathrm{C}_{4} \mathrm{H}_{9}\right)_{4} \mathrm{Br}^{[7]}$ 、 PEG-400 $0^{[8]} 、 \mathrm{InCl}_{3} \cdot 3 \mathrm{H}_{2} \mathrm{O}^{[9]} 、 \mathrm{InF}_{3}{ }^{[10]}$ 、分子碘 ${ }^{[1]]}$ 、烷基苯 磺酸 ${ }^{[2]}$ 、离子液体 ${ }^{[13,14]}$ 、铁磁流体 ${ }^{[15]}$ 等, 也有负载型催 化剂如 $\mathrm{SiO}_{2}-\mathrm{TiCl}_{4}{ }^{[16]} 、 \mathrm{Al}_{2} \mathrm{O}_{3}-\mathrm{NiCl}_{2}{ }^{[17]}$ 、硅胶- $\mathrm{NaHSO}_{4}{ }^{[18]}$ 、
SBA-15-TFE ${ }^{[19]}$ 、粘土负载钛化合物 ${ }^{[20]}$ 、高聚物催化剂 ${ }^{[21]}$ 等. 尤其是近年来，纳米材料负载催化剂在合成多取代 咪唑类化合物中得到广泛应用，如纳米硅负载有机 酸 ${ }^{[22]}$ 、水合氧化铝纳米颗粒 ${ }^{[23]}$ 、纳米晶体 $\mathrm{MgAl}_{2} \mathrm{O}_{3}{ }^{[24]}$ 、 纳米 $\mathrm{Fe}_{3} \mathrm{O}_{4}$ 负载离子液体 ${ }^{[25]}$ 等. 另外, 微波辐射 ${ }^{[25]}$ 及超 声波辐射 ${ }^{[24,26]}$ 对合成多取代咪唑也有很好的促进作用.

虽然在一锅法合成多取代咪唑类化合物方面报道 了许多新型催化剂，但不少催化剂存在制备工艺复杂、 原料不易获得、成本较高等缺点, 因而, 需要寻求一种 高效、价廉，容易制备的催化剂. $\mathrm{FeCl}_{3}$ 廉价易得，绿色 环保, 具有较强的 Lewis 酸性. $\mathrm{FeCl}_{3}$ 与活性炭、 $\mathrm{SiO}_{2}$ 、 高分子材料、离子交换树脂等负载后催化有机反应，克 服了催化剂稳定性差、不易分离纯化、不可循环使用等 缺点, 受到人们的广泛关注 ${ }^{[27 ~ 29]}$. 最近, 我们在聚苯乙 烯负载 $\mathrm{FeCl}_{3}$ 催化下, 采用一锅法高收率合成了 $\beta$-氨基

\footnotetext{
*E-mail: xiaoliwei2000@sina.com

Received June 26, 2014; revised August 8, 2014; published online August 28, 2014.

Project supported by the Natural Science Foundation of Hebei Province (No. B2013408014) and the Key Foundation of Langfang Teachers University (No. LSLZ201402).

河北省自然科学基金(No. B2013408014)和廊坊师范学院重点(No. LSLZ201402)资助项目.
} 
酮类衍生物 ${ }^{[30]}$.

本文制备了一种改性活性炭负载 $\mathrm{FeCl}_{3}$ 催化剂 $\left(\mathrm{MAC}-\mathrm{FeCl}_{3}\right)$, 通过等离子体发射光谱(ICP)及红外光谱 (IR)进行了表征. 在该催化剂作用下, 以乙酸铵为胺源, 苯偶酰与芳醛以及伯胺作用, 多组分一锅法合成了一系 列 2,4,5-三芳基咪唑和 1,2,4,5-四芳基咪唑. 反应结束后, $\mathrm{MAC}-\mathrm{FeCl}_{3}$ 催化剂经简单处理，可循环使用.

\section{1 结果与讨论}

\section{$1.1 \mathrm{MAC}-\mathrm{FeCl}_{3}$ 催化剂的表征}

自制的改性活性炭(MAC)的红外光谱中, 在 3415、 1637 和 $1087 \mathrm{~cm}^{-1}$ 处有很强的吸收峰, 这是 $\mathrm{O}-\mathrm{H}$ 的伸 缩振动、弯曲振动和 $\mathrm{C}-\mathrm{O}$ 键的伸缩振动吸收, 说明改 性活性炭中含有丰富的羟基等官能团，而普通活性炭 (AC)的红外光谱, 在这些波数处的吸收峰不明显. 改性 活性炭负载 $\mathrm{FeCl}_{3}$ 后的红外光谱, $\mathrm{C}-\mathrm{O}$ 键伸缩振动吸收 带从 $1087 \mathrm{~cm}^{-1}$ 蓝移到 $1124 \mathrm{~cm}^{-1}$ 附近, 且强度有所加 强. 这是由于 $\mathrm{C}-\mathrm{O}-\mathrm{H}$ 官能团中氧原子上的孤对电子 填充到缺电子的 $\mathrm{FeCl}_{3}$ 空轨道中, 使 $\mathrm{C}-\mathrm{O}$ 伸缩振动的 不对称性加强所致. 同时, 差基的伸缩振动也有微小变 化, 从 $3415 \mathrm{~cm}^{-1}$ 迁移到 $3431 \mathrm{~cm}^{-1}$ (图 1).

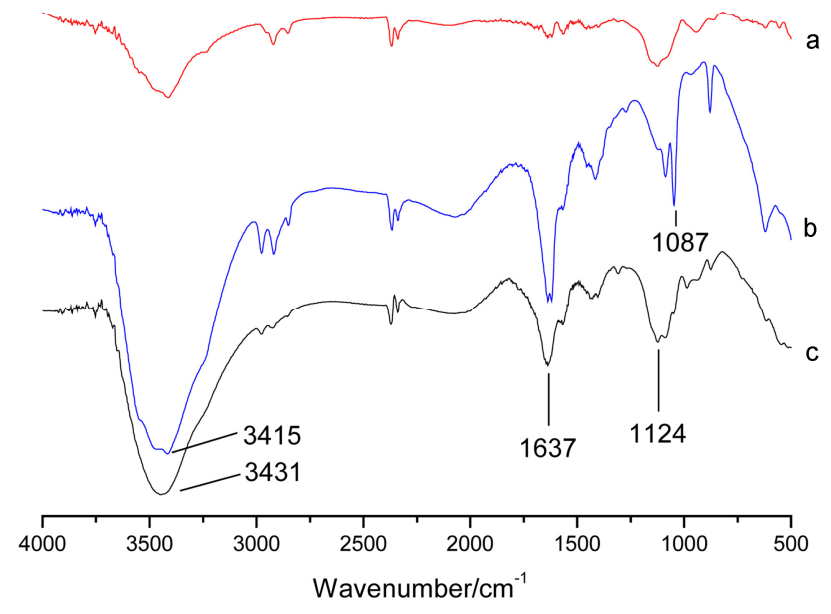

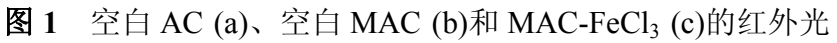
谱

Figure 1 The IR spectra of $\mathrm{AC}(\mathrm{a}), \mathrm{MAC}(\mathrm{b})$ and $\mathrm{MAC}-\mathrm{FeCl}_{3}$ (c)

通过等离子体发射光谱法(ICP)测定 $\mathrm{MAC}-\mathrm{FeCl}_{3}$ 的 支载量约为 $2.102 \mathrm{mmol} / \mathrm{g}$, 明显高于 $\mathrm{AC}-\mathrm{FeCl}_{3}$ 的支载量 $(1.640 \mathrm{mmol} / \mathrm{g})$. 因为 $\mathrm{MAC}$ 中含有丰富的羟基，与 $\mathrm{FeCl}_{3}$ 具有很好的配位能力, 因而比 $\mathrm{AC}$ 的负载能力更强, 这 是 $\mathrm{MAC}-\mathrm{FeCl}_{3}$ 具有强催化活性的重要原因.

\section{2 反应条件的优化}

1.2.1 催化剂及其用量对反应的影响

以苯偶酰 $(10 \mathrm{mmol}, 2.10 \mathrm{~g})$ 与苯甲醛 $(10 \mathrm{mmol}, 1.06$ $\mathrm{g})$ 或苯胺 $(10 \mathrm{mmol}, 0.93 \mathrm{~g})$ 之间的反应为模板，探讨一 锅法合成三苯基咪唑和四苯基咪唑的最佳反应条件 (Scheme 1). 催化剂的用量对反应结果的影响情况见表 1. 从表 1 中可以看出, 随着 $\mathrm{MAC}-\mathrm{FeCl}_{3}$ 用量增大, 三苯 基咪唑产率逐渐提高, 当 $\mathrm{MAC}^{-} \mathrm{FeCl}_{3}$ 用量达到 $0.4 \mathrm{~g}$ 时, 继续增加, 产率升高不明显. 因而, 将催化剂的用量设 定为 $0.4 \mathrm{~g}$, 即反应底物与 $\mathrm{FeCl}_{3}$ 之间的物质的量比为 $1: 0.084$. 类似地, 制备四苯基咪唑时, $\mathrm{MAC}-\mathrm{FeCl}_{3}$ 合适 的用量为 $0.5 \mathrm{~g}$, 即反应底物与 $\mathrm{FeCl}_{3}$ 之间的物质的量比 为 $1: 0.105$. 可见, 相同用量的 $\mathrm{MAC}-\mathrm{FeCl}_{3}$ 催化活性明 显高于 $\mathrm{AC}-\mathrm{FeCl}_{3}$, 原因是 $\mathrm{MAC}-\mathrm{FeCl}_{3}$ 中活性成分的含 量高于 $\mathrm{AC}_{-} \mathrm{FeCl}_{3}$.

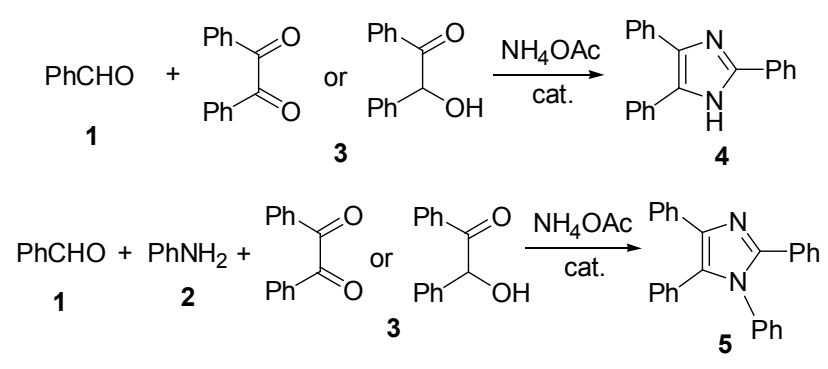

Scheme 1

表 1 催化剂及其用量对反应的影响

Table 1 Type of catalyst and catalyst loading effect on the reaction

\begin{tabular}{|c|c|c|c|c|c|}
\hline Entry & Catalyst & Catalyst/g & $\mathrm{FeCl}_{3} / \mathrm{mmol}$ & $\begin{array}{c}\text { Yield }^{a} / \% \\
\text { of } \mathbf{4 a}\end{array}$ & $\begin{array}{c}\text { Yield }^{b} / \% \\
\text { of } \mathbf{5 a}\end{array}$ \\
\hline 1 & MAC-FeCl 3 & 0.1 & 0.21 & 55.6 & 49.8 \\
\hline 2 & MAC-FeCl 3 & 0.2 & 0.42 & 68.3 & 63.3 \\
\hline 3 & MAC-FeCl 3 & 0.3 & 0.63 & 76.5 & 71.5 \\
\hline 4 & MAC-FeCl 3 & 0.4 & 0.84 & 89.4 & 80.2 \\
\hline 5 & MAC-FeCl 3 & 0.5 & 1.05 & 89.5 & 87.6 \\
\hline 6 & $\mathrm{AC}-\mathrm{FeCl}_{3}$ & 0.4 & 0.64 & 77.3 & 71.4 \\
\hline 7 & $\mathrm{AC}-\mathrm{FeCl}_{3}$ & 0.5 & 0.80 & 83.7 & 79.6 \\
\hline
\end{tabular}

${ }^{a}$ Reaction condition: benzil $10 \mathrm{mmol}$, benzaldehyde $10 \mathrm{mmol}, \mathrm{NH}_{4} \mathrm{OAc} 20$ mmol, EtOH $10 \mathrm{~mL}, 60 \mathrm{~min} .{ }^{b}$ Reaction condition: benzil $10 \mathrm{mmol}$, benzaldehyde $10 \mathrm{mmol}$, aniline $10 \mathrm{mmol}, \mathrm{NH}_{4} \mathrm{OAc} 10 \mathrm{mmol}$, DMF $10 \mathrm{~mL}, 60 \mathrm{~min}$.

\subsection{2 溶剂对反应的影响}

在合成三苯基咪唑的过程中，分别选用乙醇、二甲 基甲酰胺(DMF)、四氢呋喃(THF)、水、氯仿及甲苯等 作为溶剂, 结果发现: 高极性溶剂有利于反应的进行, 乙醇是该反应最理想的溶剂(表 2). 但在合成四取代咪 唑的过程中, 用 DMF 作溶剂产率比用乙醇高得多，这 说明形成四苯基咪唑的关键步骤需要在较高温度下进 行, 用低沸点溶剂, 反应温度不够.

\section{3 不同的底物对反应的影响}

\subsection{1 不同的醛为底物合成三芳基咪唑}

为进一步探讨 $\mathrm{MAC}-\mathrm{FeCl}_{3}$ 对合成三取代咪唑类化 
表 2 溶剂对反应的影响

Table 2 Solvent effect on the reaction

\begin{tabular}{ccccc}
\hline Entry & Solvent & Time/min & Yield $^{a} / \%$ of $\mathbf{4 a}$ & Yield $^{b} / \%$ of $\mathbf{5 a}$ \\
\hline 1 & EtOH & 60 & 89.4 & 73.8 \\
2 & THF & 90 & 54.5 & 47.6 \\
3 & $\mathrm{DMF}$ & 75 & 87.8 & 87.6 \\
4 & $\mathrm{H}_{2} \mathrm{O}$ & 120 & No reaction & No reaction \\
5 & $\mathrm{CHCl}_{3}$ & 120 & Trace & Trace \\
6 & Toluene & 120 & Trace & Trace \\
\hline
\end{tabular}

${ }^{a}$ Reaction condition: benzil $10 \mathrm{mmol}$, benzaldehyde $10 \mathrm{mmol}, \mathrm{NH}_{4} \mathrm{OAc} 20$ mmol, $\mathrm{MAC}-\mathrm{FeCl}_{3} 0.4$ g. ${ }^{b}$ Reaction condition: benzil $10 \mathrm{mmol}$, benzaldehyde $10 \mathrm{mmol}$, aniline $10 \mathrm{mmol}, \mathrm{NH}_{4} \mathrm{OAc} 10 \mathrm{mmol}, \mathrm{MAC}^{-\mathrm{FeCl}_{3}} 0.5 \mathrm{~g}$.

合物的促进作用, 在优化的实验条件下，换用含不同取 代基的芳醛进行反应(Eq. 1, 结果见表 3). 结果表明, 无 论以含有吸电子基团还是供电子基团的芳醛为原料, 均 能得到满意的结果. 苯环上带供电子基团的芳醛为原料 时, 相应的三取代咪唑的收率略高于苯环上带吸电子基 团的情况. 选用脂肪醛进行上述实验, 产率比芳醛低很 多或根本观察不到产物.

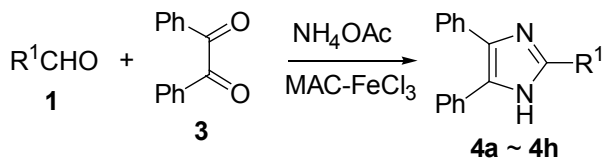

表 $3 \mathrm{MAC}-\mathrm{FeCl}_{3}$ 催化合成三芳基咪唑 4

Table 3 Synthesis of triarylimidazoles 4 catalyzed by MAC$\mathrm{FeCl}_{3}{ }^{a}$

\begin{tabular}{clcc}
\hline Product & \multicolumn{1}{c}{$\mathrm{R}^{1}$} & Time $/$ min & Yield/\% \\
\hline 4a & $\mathrm{Ph}$ & 75 & 89.4 \\
$\mathbf{4 b}$ & $p-\mathrm{H}_{3} \mathrm{CC}_{6} \mathrm{H}_{4}$ & 75 & 87.6 \\
$\mathbf{4 c}$ & $p-\mathrm{H}_{3} \mathrm{COC}_{6} \mathrm{H}_{4}$ & 75 & 91.3 \\
$\mathbf{4 d}$ & $p-\mathrm{ClC}_{6} \mathrm{H}_{4}$ & 75 & 83.2 \\
$\mathbf{4 e}$ & $p-\mathrm{HOC}_{6} \mathrm{H}_{4}$ & 70 & 80.7 \\
$\mathbf{4 f}$ & $p-\mathrm{BrC}_{6} \mathrm{H}_{4}$ & 75 & 83.7 \\
$\mathbf{4 g}$ & $p-\mathrm{O}_{2} \mathrm{NC}_{6} \mathrm{H}_{4}$ & 65 & 79.1 \\
$\mathbf{4 h}$ & $m-\mathrm{O}_{2} \mathrm{NC}_{6} \mathrm{H}_{4}$ & 65 & 82.5 \\
\hline
\end{tabular}

${ }^{a}$ Reaction condition: benzyl $10 \mathrm{mmol}$, benzaldehyde $10 \mathrm{mmol}, \mathrm{NH}_{4} \mathrm{OAc} 20$

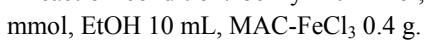

\subsection{2 不同的醛和胺为底物合成四芳基咪唑}

类似地, 在优化的实验条件下, 以 $\mathrm{MAC}-\mathrm{FeCl}_{3}$ 为催 化剂, 考察苯环上含不同取代基的芳醛及芳胺为原料合 成四芳基咪唑类化合物的情况(Eq. 2), 结果见表 4. 从 表 4 可以看出, $\mathrm{MAC}-\mathrm{FeCl}_{3}$ 对含有不同取代基的四芳基 咪唑类化合物的合成均具有较好的催化作用. 相对于芳 胺, 脂肪胺碱性较强(超过氨水), 长时间回流反应会导 致催化剂中部分氯化铁水解.

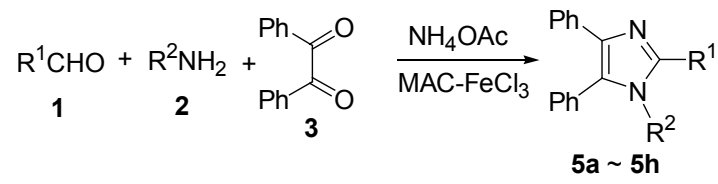

表 $4 \mathrm{MAC}-\mathrm{FeCl}_{3}$ 催化合成四芳基咪唑 $\mathbf{5}^{a}$

Table 4 Synthesis of tetraarylimidazoles 5 catalyzed by MAC$\mathrm{FeCl}_{3}$

\begin{tabular}{|c|c|c|c|c|}
\hline Product & $\mathrm{R}^{1}$ & $\mathrm{R}^{2}$ & Time/min & Yield/\% \\
\hline $5 \mathbf{a}$ & $\mathrm{Ph}$ & $\mathrm{Ph}$ & 90 & 87.6 \\
\hline $5 b$ & $p-\mathrm{H}_{3} \mathrm{CC}_{6} \mathrm{H}_{4}$ & $\mathrm{Ph}$ & 90 & 85.2 \\
\hline $5 c$ & $p-\mathrm{H}_{3} \mathrm{COC}_{6} \mathrm{H}_{4}$ & $\mathrm{Ph}$ & 90 & 87.5 \\
\hline $5 d$ & $p-\mathrm{ClC}_{6} \mathrm{H}_{4}$ & $\mathrm{Ph}$ & 120 & 83.6 \\
\hline $5 e$ & $p-\mathrm{O}_{2} \mathrm{NC}_{6} \mathrm{H}_{4}$ & $\mathrm{Ph}$ & 90 & 90.5 \\
\hline $5 f$ & $p-\mathrm{H}_{3} \mathrm{CC}_{6} \mathrm{H}_{4}$ & $p-\mathrm{H}_{3} \mathrm{CC}_{6} \mathrm{H}_{4}$ & 90 & 82.4 \\
\hline $5 g$ & $p-\mathrm{O}_{2} \mathrm{NC}_{6} \mathrm{H}_{4}$ & $p-\mathrm{H}_{3} \mathrm{CC}_{6} \mathrm{H}_{4}$ & 120 & 80.8 \\
\hline $5 \mathrm{~h}$ & $p-\mathrm{ClC}_{6} \mathrm{H}_{4}$ & $p-\mathrm{ClC}_{6} \mathrm{H}_{4}$ & 120 & 84.9 \\
\hline
\end{tabular}

mmol, $\mathrm{NH}_{4} \mathrm{OAc} 10 \mathrm{mmol}$, DMF $10 \mathrm{~mL}, \mathrm{MAC}-\mathrm{FeCl}_{3} 0.5 \mathrm{~g}$.

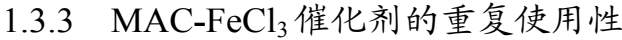

以苯甲醛、苯偶酰和醋酸铵作用制备三苯基咪唑的 反应为例, 研究 $\mathrm{MAC}-\mathrm{FeCl}_{3}$ 催化剂的重复使用性能(表 5): 催化剂使用 4 次后仍保持很高的活性, 但收率略有 下降. 通过对比新制备和回收(循环 2 次)的催化剂的红 外光谱发现, 回收的 $\mathrm{MAC}-\mathrm{FeCl}_{3}$ 的红外光谱中, $\mathrm{C}-\mathrm{O}$ 键伸缩振动吸收带红移到 $1116 \mathrm{~cm}^{-1}$, 与新制 MAC- $-\mathrm{FeCl}_{3}\left(1124 \mathrm{~cm}^{-1}\right)$ 相比, 发生了红移, 这可能是由 于催化剂中 $\mathrm{FeCl}_{3}$ 含量略有下降所致. 但与空白 $\mathrm{MAC}$ 的 相应吸收峰 $\left(1087 \mathrm{~cm}^{-1}\right)$ 相比, 有所蓝移, 说明回收的催 化剂中仍含有较多的 $\mathrm{FeCl}_{3}$. 同时, 羟基的伸缩振动也 有微小变化, 从 $3431 \mathrm{~cm}^{-1}$ 迁移到 $3425 \mathrm{~cm}^{-1}$ (图 2). 进 一步通过 ICP 法测定循环使用的 $\mathrm{MAC}-\mathrm{FeCl}_{3}$ 中 $\mathrm{FeCl}_{3}$ 的 含量发现, 随着循环使用次数的增加, 催化剂中 $\mathrm{FeCl}_{3}$ 的含量略有降低, 因而导致其催化活性有所降低, 与红 外光谱测定的结果一致.

表 $5 \mathrm{MAC}-\mathrm{FeCl}_{3}$ 催化剂循环使用情况

Table 5 The recycling behavior of $\mathrm{MAC}-\mathrm{FeCl}_{3}$

\begin{tabular}{lcccc}
\hline Recycle number & Fresh & 1 & 2 & 3 \\
\hline $\mathrm{FeCl}_{3}{ }^{a}$ in Cat./(mmol•g & \\
& 2.102 & 1.931 & 1.803 & 1.726 \\
Yield/\% & 89.4 & 87.2 & 85.4 & 82.3 \\
\hline${ }^{a} \mathrm{FeCl}_{3}$ contents in fresh catalyst or recycled catalyst were determined by ICP.
\end{tabular}

\section{2 实验部分}

\section{1 仪器与试剂}

所用的实验原料中, 改性活性炭及其负载 $\mathrm{FeCl}_{3}$ 催 化剂自制, 其他药品均为采购的分析纯试剂, 使用前未 做进一步处理. 产物的熔点由XT4A型显微熔点仪测定, 温度未校正; 红外光谱由 IR Prestige-21 傅里叶变换红 外光谱仪测定, $\mathrm{KBr}$ 压片; $\mathrm{MAC}-\mathrm{FeCl}_{3}$ 催化剂中 $\mathrm{FeCl}_{3}$ 的 含量由 IRIS-Advantage 全谱直读等离子体发射光谱仪测 定; 核磁共振谱由 AVANCE 400 型核磁共振波谱仪测 定, $\mathrm{CDCl}_{3}$ 为溶剂. 


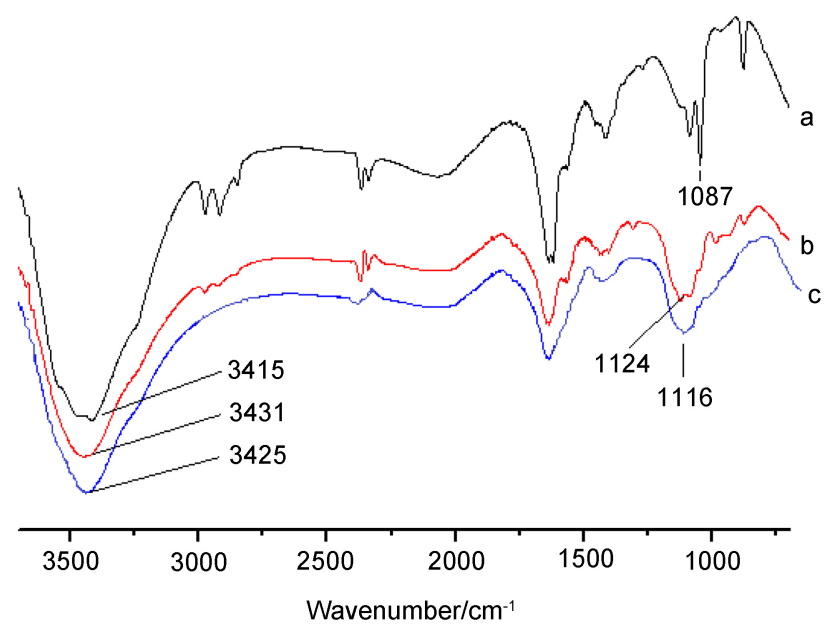

图 2 空白 $\mathrm{MAC}(\mathrm{a})$ 、新制 $\mathrm{MAC}-\mathrm{FeCl}_{3}$ (b) 和回收 $\mathrm{MAC}-\mathrm{FeCl}_{3}$ (c)的红外光谱

Figure 2 The IR spectra of blank MAC (a), fresh MAC-FeCl (b) and recovery $\mathrm{MAC}-\mathrm{FeCl}_{3}$ (c)

\section{2 改性活性炭的制备}

取 $20 \mathrm{~g}$ 洗刷干净的花生壳粉碎, 与适量浓硫酸混合 均匀, 室温下摚拌 $24 \mathrm{~h}$. 用 $\mathrm{NaOH}$ 溶液调至中性, 抽滤, 滤饼分别用水、乙醇及丙酮洗涤, 于 $130{ }^{\circ} \mathrm{C}$ 烘干, 冷却 后充分研磨，过篮(100 150 目), 得改性活性炭(MAC).

\section{$2.3 \mathrm{MAC}-\mathrm{FeCl}_{3}$ 催化剂的制备}

称量 $10 \mathrm{~g}$ 上步所得 $\mathrm{MAC}$ 于烧瓶中, 将 $10 \mathrm{~g}$ 无水 $\mathrm{FeCl}_{3}$ 溶于乙醇后分批加入烧瓶中, 回流 $4 \mathrm{~h}$. 蒸除溶剂, 反应体系冷却至室温, 产物分别用水、乙醇和丙酮充分 洗涤, 于 $120{ }^{\circ} \mathrm{C}$ 下活化 $4 \mathrm{~h}$, 得 $\mathrm{MAC}-\mathrm{FeCl}_{3}$ 催化剂. 通 过 ICP 法测定 $\mathrm{MAC}-\mathrm{FeCl}_{3}$ 中铁的含量, 经计算催化剂的 支载量为 $2.102 \mathrm{mmol} / \mathrm{g}$.

\section{$2.4 \mathrm{AC}-\mathrm{FeCl}_{3}$ 催化剂的制备}

称量 $10 \mathrm{~g}$ 普通试剂级活性炭 $\mathrm{AC}$ 于烧瓶中, 经过与 制备 $\mathrm{MAC}-\mathrm{FeCl}_{3}$ 相同的步骤, 得 $\mathrm{AC}-\mathrm{FeCl}_{3}$ 催化剂. 通过 ICP 法测定 $\mathrm{AC}-\mathrm{FeCl}_{3}$ 中铁的含量, 经计算其支载量为 $1.640 \mathrm{mmol} / \mathrm{g}$.

\section{$2.5 \mathrm{MAC}-\mathrm{FeCl}_{3}$ 催化剂促进三芳基咪唑的合成通法}

$50 \mathrm{~mL}$ 圆底烧瓶中加入芳醛 $10 \mathrm{mmol}$, 苯偶酰 10 $\mathrm{mmol}$, 乙酸铵 $20 \mathrm{mmol}$ 和 $10 \mathrm{~mL}$ 乙醇, 摚拌使之溶解,

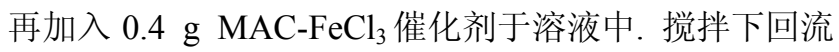
60 120 min, 反应过程由薄层色谱(TLC)跟踪. 待反应 完成后, 趁热过滤. 用乙醇洗涤滤饼, 洗涤液与滤液合 并. 滤液经浓缩、冷却, 有大量固体析出, 用乙醇重结晶 得纯品. 经过充分洗涤的滤渣, 在 $100{ }^{\circ} \mathrm{C}$ 温度下烘干即 可重复使用. 所得三芳基咪唑均是已知化合物, 其波谱 数据如下:
2,4,5-三苯基-1H-咪唑(4a)：白色固体，m.p. 279 $280{ }^{\circ} \mathrm{C}$ (lit. $\left.{ }^{[4]} 277 \sim 278{ }^{\circ} \mathrm{C}\right) ;{ }^{1} \mathrm{H}$ NMR (400 MHz, $\mathrm{CDCl}_{3}$ ) $\delta: 7.28 \sim 7.44(\mathrm{~m}, 10 \mathrm{H}), 7.60(\mathrm{~d}, J=8.0 \mathrm{~Hz}, 4 \mathrm{H}), 8.10(\mathrm{~d}$, $J=8.2 \mathrm{~Hz}, 1 \mathrm{H})$; IR (KBr) $v: 3426,3052,1660,1550,1489$ $\mathrm{cm}^{-1}$.

2-(4-甲基苯基)-4,5-二苯基- $1 H$-咪唑(4b)：白色固 体, m.p. $232 \sim 233{ }^{\circ} \mathrm{C}$ (lit. ${ }^{[4]} 230 \sim 232{ }^{\circ} \mathrm{C}$ ); ${ }^{1} \mathrm{H}$ NMR $\left(400 \mathrm{MHz}, \mathrm{CDCl}_{3}\right) \delta: 2.39(\mathrm{~s}, 3 \mathrm{H}), 7.23 \sim 7.35(\mathrm{~m}, 8 \mathrm{H})$, $7.51 \sim 7.55(\mathrm{~m}, 4 \mathrm{H}), 7.78(\mathrm{~d}, J=8.0 \mathrm{~Hz}, 2 \mathrm{H})$; IR $(\mathrm{KBr}) v$ : $3450,2965,1600,1585 \mathrm{~cm}^{-1}$.

2-(4-甲氧基苯基)-4,5-二苯基- $1 H$-咪唑(4c)：白色固 体, m.p. $227 \sim 228{ }^{\circ} \mathrm{C}$ (lit. ${ }^{[4]} 229 \sim 230{ }^{\circ} \mathrm{C}$ ); ${ }^{1} \mathrm{H}$ NMR $\left(400 \mathrm{MHz}, \mathrm{CDCl}_{3}\right) \delta: 3.80(\mathrm{~s}, 3 \mathrm{H}), 6.90(\mathrm{~d}, J=8.8 \mathrm{~Hz}$, 2H), $7.23 \sim 7.48(\mathrm{~m}, 6 \mathrm{H}), 7.49(\mathrm{~d}, J=7.0 \mathrm{~Hz}, 4 \mathrm{H}), 7.91(\mathrm{~d}$, $J=8.8 \mathrm{~Hz}, 2 \mathrm{H}) ; \mathrm{IR}(\mathrm{KBr}) v: 3400,3060,2945,1611,1490$ $\mathrm{cm}^{-1}$.

2-(4-氯苯基)-4,5-二苯基- $1 H$-咪唑(4d)：白色固体, m.p. $261 \sim 262{ }^{\circ} \mathrm{C}$ (lit. $\left.{ }^{[4]} 260 \sim 262{ }^{\circ} \mathrm{C}\right) ;{ }^{1} \mathrm{H}$ NMR $(400$ $\left.\mathrm{MHz}, \mathrm{CDCl}_{3}\right) \delta: 7.25 \sim 7.31(\mathrm{~m}, 8 \mathrm{H}), 7.48(\mathrm{~d}, J=7.6 \mathrm{~Hz}$, $4 \mathrm{H}), 7.79(\mathrm{~d}, J=8.2 \mathrm{~Hz}, 2 \mathrm{H})$; IR (KBr) $v: 3412,1502$, $1632,1502 \mathrm{~cm}^{-1}$.

4-(4,5-二苯基-1H-咪唑基-2)-苯酚(4e): 白色固体, m.p. 266 267 ${ }^{\circ} \mathrm{C}$ (lit. ${ }^{[4]} 268 \sim 269{ }^{\circ} \mathrm{C}$ ); ${ }^{1} \mathrm{H}$ NMR $(400$ $\left.\mathrm{MHz}, \mathrm{CDCl}_{3}\right) \delta: 6.78(\mathrm{~d}, J=7.8 \mathrm{~Hz}, 1 \mathrm{H}), 7.48 \sim 7.53(\mathrm{~m}$, $5 \mathrm{H}), 7.15 \sim 7.29(\mathrm{~m}, 6 \mathrm{H}), 7.98(\mathrm{~d}, J=8.0 \mathrm{~Hz}, 2 \mathrm{H}), 9.97$ (brs, 1H); IR (KBr) v: 3434, 1638, 1605, 1539, $1493 \mathrm{~cm}^{-1}$.

2-(4-溴苯基)-4,5-二苯基- $1 H$-咪唑(4f)：白色固体, m.p. $263 \sim 264{ }^{\circ} \mathrm{C}$ (lit. $\left.{ }^{[4]} 260 \sim 262{ }^{\circ} \mathrm{C}\right) ;{ }^{1} \mathrm{H}$ NMR $(400$ $\left.\mathrm{MHz}, \mathrm{CDCl}_{3}\right) \delta: 7.25 \sim 7.29(\mathrm{~m}, 8 \mathrm{H}), 7.31 \sim 7.52(\mathrm{~m}, 4 \mathrm{H})$, $7.76(\mathrm{~d}, J=8.2 \mathrm{~Hz}, 2 \mathrm{H})$; IR (KBr) $v: 3425,3058,1484$, $1630 \mathrm{~cm}^{-1}$.

2-(4-硝基苯基)-4,5-二苯基- $1 H$-咪唑(4g)：白色固 体, m.p. $198 \sim 199{ }^{\circ} \mathrm{C}$ (lit. ${ }^{[4]} 200 \sim 202{ }^{\circ} \mathrm{C}$ ); ${ }^{1} \mathrm{H}$ NMR $\left(400 \mathrm{MHz}, \mathrm{CDCl}_{3}\right) \delta: 7.26 \sim 7.49(\mathrm{~m}, 6 \mathrm{H}), 7.69$ (d, $J=7.6$ $\mathrm{Hz}, 2 \mathrm{H}), 7.74$ (d, $J=7.8 \mathrm{~Hz}, 4 \mathrm{H}), 8.14$ (d, $J=7.2 \mathrm{~Hz}, 2 \mathrm{H})$; IR (KBr) $v: 3456,1640,1589,1523,1348 \mathrm{~cm}^{-1}$.

2-(3-硝基苯基)-4,5-二苯基- $1 H$-咪唑(4h): 白色固 体, m.p. $>300{ }^{\circ} \mathrm{C}$ (lit. $\left.{ }^{[8]} 303 \sim 304{ }^{\circ} \mathrm{C}\right) ;{ }^{1} \mathrm{H}$ NMR $(400$ $\left.\mathrm{MHz}, \mathrm{CDCl}_{3}\right) \delta: 7.27 \sim 7.67(\mathrm{~m}, 12 \mathrm{H}), 7.95(\mathrm{~d}, J=7.6 \mathrm{~Hz}$, $1 \mathrm{H}), 8.12(\mathrm{~d}, J=7.5 \mathrm{~Hz}, 1 \mathrm{H})$; IR (KBr) $v: 3452,1650$, $1590,1524,1350 \mathrm{~cm}^{-1}$.

\section{$2.6 \mathrm{MAC}-\mathrm{FeCl}_{3}$ 催化剂促进四芳基咪唑的合成通法}

$50 \mathrm{~mL}$ 圆底烧瓶中加入芳醛 $10 \mathrm{mmol}$, 芳伯胺 10

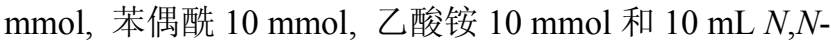
二甲基甲酰胺(DMF), 再加入 $0.5 \mathrm{~g} \mathrm{MAC}-\mathrm{FeCl}_{3}$ 于体系 
中, 搅拌下回流 $90 \sim 150 \mathrm{~min}$. 反应过程及反应完成后 产品处理方法与三芳基咪唑的处理方法类似. 所得四芳 基咪唑化合物均是已知化合物，其波谱数据如下：

1,2,4,5-四苯基咪唑(5a)：白色固体, m.p. 219 220 ${ }^{\circ} \mathrm{C}$ (lit. ${ }^{[8]} 219 \sim 220{ }^{\circ} \mathrm{C}$ ); ${ }^{1} \mathrm{H}$ NMR (400 MHz, $\left.\mathrm{CDCl}_{3}\right) \delta$ : $7.13 \sim 7.16(\mathrm{~m}, 5 \mathrm{H}), 7.33 \sim 7.36(\mathrm{~m}, 6 \mathrm{H}), 7.45 \sim 7.50(\mathrm{~m}$, 4H), 8.00 8.02 (m, 5H); IR (KBr) v: 3062, 1605, 1580, $1498 \mathrm{~cm}^{-1}$.

2-(4-甲基苯基)-1,4,5-三苯基咪唑(5b): 白色固体, m.p. $185 \sim 187{ }^{\circ} \mathrm{C}$ (lit. $\left.{ }^{[10]} 186 \sim 188{ }^{\circ} \mathrm{C}\right) ;{ }^{1} \mathrm{H}$ NMR $(400$ $\left.\mathrm{MHz}, \mathrm{CDCl}_{3}\right) \delta: 2.26(\mathrm{~s}, 3 \mathrm{H}), 7.07(\mathrm{~d}, J=8.0 \mathrm{~Hz}, 2 \mathrm{H})$, $7.16 \sim 7.18(\mathrm{~m}, 1 \mathrm{H}), 7.23 \sim 7.25(\mathrm{~m}, 6 \mathrm{H}), 7.27 \sim 7.32(\mathrm{~m}$, $8 \mathrm{H}), 7.51(\mathrm{~d}, J=8.0 \mathrm{~Hz}, 2 \mathrm{H})$; IR $(\mathrm{KBr}) v: 3055,2935$, $1590,1577,1492 \mathrm{~cm}^{-1}$.

2-(4-甲氧基苯基)-1,4,5-三苯基咪唑(5c)：白色固体， m.p. $177 \sim 178{ }^{\circ} \mathrm{C}$ (lit. $\left.{ }^{[8]} 174 \sim 175{ }^{\circ} \mathrm{C}\right) ;{ }^{1} \mathrm{H}$ NMR $(400$ $\left.\mathrm{MHz}, \mathrm{CDCl}_{3}\right) \delta: 3.70(\mathrm{~s}, 3 \mathrm{H}), 6.80(\mathrm{~d}, J=8.0 \mathrm{~Hz}, 2 \mathrm{H})$, $7.16(\mathrm{~d}, J=8.4 \mathrm{~Hz}, 1 \mathrm{H}), 7.18 \sim 7.20(\mathrm{~m}, 3 \mathrm{H}), 7.21 \sim 7.31$ (m, $5 \mathrm{H}), 7.40 \sim 7.41(\mathrm{~m}, 3 \mathrm{H}), 7.42 \sim 7.46(\mathrm{~m}, 3 \mathrm{H}), 7.48(\mathrm{~d}$, $J=8.0 \mathrm{~Hz}, 2 \mathrm{H}) ; \mathrm{IR}(\mathrm{KBr}) v: 3043,2917,1590,1565,1478$ $\mathrm{cm}^{-1}$.

2-(4-氯苯基)-1,4,5-三苯基咪唑(5d): 白色固体, m.p. $154 \sim 155{ }^{\circ} \mathrm{C}$ (lit. $\left.{ }^{[8]} 157 \sim 158{ }^{\circ} \mathrm{C}\right) ;{ }^{1} \mathrm{H}$ NMR $(400 \mathrm{MHz}$, $\left.\mathrm{CDCl}_{3}\right) \delta: 6.76(\mathrm{~d}, J=6.2 \mathrm{~Hz}, 2 \mathrm{H}), 7.14 \sim 7.20(\mathrm{~m}, 6 \mathrm{H})$, $7.30(\mathrm{~d}, J=5.6 \mathrm{~Hz}, 2 \mathrm{H}), 7.40 \sim 7.47(\mathrm{~m}, 7 \mathrm{H}), 7.58(\mathrm{~d}, J=$ $7.4 \mathrm{~Hz}, 2 \mathrm{H})$; IR (KBr) v: 3064, 1592, 1480, $1425 \mathrm{~cm}^{-1}$.

2-(4-硝基苯基)-1,4,5-三苯基咪唑(5e): 白色固体. m.p. $190 \sim 192{ }^{\circ} \mathrm{C}$ (lit. $\left.{ }^{[8]} 191 \sim 193{ }^{\circ} \mathrm{C}\right) ;{ }^{1} \mathrm{H}$ NMR $(400$ $\left.\mathrm{MHz}, \mathrm{CDCl}_{3}\right) \delta: 7.36 \sim 7.46(\mathrm{~m}, 9 \mathrm{H}), 7.57(\mathrm{~m}, 4 \mathrm{H}), 8.07$ (d, $J=8.2 \mathrm{~Hz}, 2 \mathrm{H}), 8.29$ (d, $J=8.0 \mathrm{~Hz}, 2 \mathrm{H}), 8.40$ (d, $J=$ $8.2 \mathrm{~Hz}, 2 \mathrm{H}$ ); IR (KBr) v: 3054, 1584, 1487, $1432 \mathrm{~cm}^{-1}$.

4,5-二苯基-1,2-二(4-甲基苯基)咪唑(5f): 白色固体, m.p. $193 \sim 194{ }^{\circ} \mathrm{C}$ (lit. $\left.{ }^{[8]} 197 \sim 198{ }^{\circ} \mathrm{C}\right) ;{ }^{1} \mathrm{H}$ NMR $(400$ $\left.\mathrm{MHz}, \mathrm{CDCl}_{3}\right) \delta: 2.25(\mathrm{~s}, 6 \mathrm{H}), 7.08 \sim 7.11(\mathrm{~m}, 6 \mathrm{H}), 7.16 \sim$ $7.24(\mathrm{~m}, 5 \mathrm{H}), 7.27 \sim 7.30(\mathrm{~m}, 5 \mathrm{H}), 7.47(\mathrm{~d}, J=6.0 \mathrm{~Hz}$, 2H); IR (KBr) v: 3050, 2935, 1595, 1508, 1473, 1440 $\mathrm{cm}^{-1}$.

2-(4-硝基苯基)-4,5-二苯基-1-(4-甲基苯基)咪唑 (5g): 白色固体, m.p. $220 \sim 221{ }^{\circ} \mathrm{C}$ (lit. ${ }^{[8]} 218 \sim 219{ }^{\circ} \mathrm{C}$ ); ${ }^{1} \mathrm{H}$ NMR $\left(400 \mathrm{MHz}, \mathrm{CDCl}_{3}\right) \delta: 2.28(\mathrm{~s}, 3 \mathrm{H}), 7.15 \sim 7.32$ $(\mathrm{m}, 12 \mathrm{H}), 7.51(\mathrm{~d}, J=6.8 \mathrm{~Hz}, 2 \mathrm{H}), 7.63(\mathrm{~d}, J=7.4 \mathrm{~Hz}$, $2 \mathrm{H}), 8.14$ (d, $J=7.6 \mathrm{~Hz}, 2 \mathrm{H})$; IR (KBr) v: 3034, 2975, $1591,1507,1334 \mathrm{~cm}^{-1}$.

1,2-二(4-氯苯基)-4,5-二苯基咪唑(5h): 白色固体, m.p. $194 \sim 195{ }^{\circ} \mathrm{C}$ (lit. $\left.{ }^{[8]} 198 \sim 200{ }^{\circ} \mathrm{C}\right) ;{ }^{1} \mathrm{H}$ NMR $(400$
$\left.\mathrm{MHz}, \mathrm{CDCl}_{3}\right) \delta: 7.18 \sim 7.19(\mathrm{~m}, 1 \mathrm{H}), 7.23 \sim 7.26(\mathrm{~m}, 4 \mathrm{H})$, $7.29 \sim 7.40(\mathrm{~m}, 11 \mathrm{H}), 7.50(\mathrm{~d}, J=7.0 \mathrm{~Hz}, 2 \mathrm{H})$; IR $(\mathrm{KBr}) v$ : $3065,1596,1499,1411 \mathrm{~cm}^{-1}$.

\section{3 结论}

综上所述，以自制的新型改性活性炭负载氯化铁为 催化剂, 以乙酸铵为氮源, 苯偶酰与芳醛以及伯胺作用, 经过多组分一锅法合成了一系列 2,4,5-三芳基咪唑和 1,2,4,5-四芳基咪唑. 与其他催化剂相比, $\mathrm{MAC}-\mathrm{FeCl}_{3}$ 催 化剂制备简单，成本低廉，催化效率高，反应条件温和， 对环境友好, 可重复使用, 反应后处理简单. 因而, 本 文发展了一种合成多芳基取代咪唑类化合物的一条方 便有效的方法，同时 $\mathrm{MAC}-\mathrm{FeCl}_{3}$ 也可作为一种潜在的 实用型催化剂应用于其他化合物的合成中.

辅助材料(Supporting Information) 16 种产物的核磁 共振氢谱原图。这些材料可以免费从本刊网站 (http://sioc-journal.cn/)上下载.

\section{References}

[1] Cozzi, P.; Carganico, G.; Fusar, D.; Grossoni, M.; Menichincheri, M.; Pinciroli, V.; Tonani, R.; Vaghi, F.; Salvati, P. J. Med. Chem. 1993, 36, 2964.

[2] Wang, L.; Woods, K. W.; Li, Q.; Barr, K. J.; McCroskey, R. W.; Hannick, S. M.; Gherke, L.; Credo, R. B.; Hui, Y. H.; Marsh, K.; Warner, R.; Lee, J. Y.; Zielinsky-Mozng, N.; Frost, D.; Rosenberg, S. H.; Sham, H. L. J. Med. Chem. 2002, 45, 1697.

[3] Dupont, J.; de Souza, R. F.; Suarez, P. A. Z. Chem. Rev. 2002, 102, 3667.

[4] Joshi, R. S.; Mundhane, P. G.; Shaikh, M. U.; Kale, R. P.; Gill, C. H. Chin. Chem. Lett. 2010, 21, 429.

[5] Sangshetti, J. N.; Kokare, N. D.; Kotharkar, S. A.; Shinde, D. B. Chin. Chem. Lett. 2008, 19, 762.

[6] Heravi, M. M.; Derikvand, F.; Bamoharram, F. F. J. Mol. Catal. A: Chem. 2007, 263, 112.

[7] Chary, M. V.; Keerthysri, N. C.; Vupallapati, S. V. N. Catal. Commun. 2008, 9, 2013.

[8] Wang, X. C.; Gong, H. P.; Quan, Z. J.; Li, L.; Ye, H. L. Chin. Chem. Lett. 2009, 20, 44.

[9] Sharma, S. D.; Hazarika, P.; Konwar, D. Tetrahedron Lett. 2008 , 49, 2216.

[10] Karunakaran, C.; Jayabharathi, J.; Kalaiarasi, V.; Jayamoorthy, K. Spectrochim. Acta, Part A 2014, 120, 84.

[11] Xue, W.-J.; Li, H.-Z.; Gao, F.-F.; Wu, A. Tetrahedron 2014, 70, 239.

[12] Das, B.; Kashanna, J.; Kumar, R. A.; Jangili, P. Monatsh. Chem. 2013, 144, 223.

[13] Zolfigol, M. A.; Khazaei, A.; Moosavi-Zare, A. R.; Zare, A.; Asgari, Z.; Khakyzadeh, V.; Hasaninejad, A. J. Ind. Eng. Chem. 2013, 19,721 .

[14] MaGee, D. I.; Bahramnejad, M.; Dabiri, M. Tetrahedron Lett. 2013, $54,2591$.

[15] Aziizi, N.; Manochehri, Z.; Nahayi, A.; Torkashvand, S. J. Mol. Liq. 2014, 196, 153.

[16] Safari, J.; Khalili, S. D.; Banitaba, S. H. Synth. Commun. 2011, 41, 
2359.

[17] Heravi, M. M.; Bakhtiari, K.; Oskooie, H. A.; Taheri, S. J. Mol. Catal. A: Chem. 2007, 263, 279.

[18] Karimi, A. R.; Alimohammadi, Z.; Azizian, J.; Mohammadi, A. A.; Mohammadizadeh, M. R. Catal. Commun. 2006, 7, 728.

[19] Rostamnia, S.; Zabardasti, A. J. Fluorine Chem. 2012, 144, 69.

[20] Kannan, V.; Sreekumar, K. J. Mol. Catal. A: Chem. 2013, 376, 34.

[21] Mohammadi, A.; Keshvari, H.; Sandaroos, R.; Maleki, B.; Rouhi, H.; Moradi, H.; Sepehr, Z.; Damavandi, S. Appl. Catal., A 2012, $429 \sim 430,73$.

[22] Ray, S.; Das, P.; Bhaumik, A.; Dutta, A.; Mukhopadhyay, C. Appl. Catal., A 2013, 458, 183.

[23] Keivanloo, A.; Bakherad, M.; Imanifar, E.; Mirzaee, M. Appl. Catal., A 2013, 467, 291.

[24] Safari, J.; Gandomi-Ravandi, S.; Akbari, Z. J. Adv. Res. 2013, 4,
509.

[25] Safari, J.; Zarnegar, Z. C. R. Chim. 2013, 16, 920.

[26] Safari, J.; Zarnegar, Z. Ultrason. Sonochem. 2013, 20, 740.

[27] Zheng, C. W.; Chai, Z.; Zhao, G. Prog. Chem. 2010, 22, 1442 (in Chinese).

(郑昌武, 柴卓, 赵刚, 化学进展, 2010, 22, 1442.)

[28] Bolm, C.; Legros, J.; Paih, J. L.; Zani, L. Chem. Rev. 2004, 104, 6217.

[29] Song, Y.; Tang, X. S.; Hou, X. M.; Bai, Y. J. Chin. J. Org. Chem. 2013, 33, 76 (in Chinese). (宋阳, 唐雪松, 侯晓萌, 白银娟, 有机化学, 2013, 33, 76.)

[30] Xiao, L. W.; Kong, J.; Zhou, Q. X.; Hu, Y. L. Chin. J. Appl. Chem. 2014, 31, 715 (in Chinese).

(肖立伟, 孔洁, 周秋香, 胡悦立, 应用化学, 2014, 31, 715.) 